\title{
OBJECT-LEVEL CHANGE DETECTION BASED ON HIGH- RESOLUTION REMOTE-SENSING IMAGES AND ITS APPLICATION IN JAPANESE EARTHQUAKE ON MARCH 11, 2011
}

\author{
Sun Kaimin ${ }^{\mathrm{a}}$, Li Deren ${ }^{\mathrm{a}^{*}}$, Sui Haigang ${ }^{\mathrm{a}}$, Liu Junyi ${ }^{\mathrm{a}}$, Ma Guorui ${ }^{\mathrm{a}}$ \\ ${ }^{\text {a }}$ State Key Laboratory of Information Engineering in Surveying, Mapping and Remote Sensing, Wuhan \\ University, Wuhan 430079, China
}

Commission VII, WG V/5

KEY WORDS: Object-level, High-resolution images, Change detection, Multi-scale segmentation, Change vector analysis

\begin{abstract}
:
In accordance with the characteristics of change detection based on high-resolution remote-sensing images, this paper has put forward an object-level change detection method that is based on multi-feature integration and can take into account the properties of different types of object. This method classifies the most essential change information in applications into artificial objects related change information, water-related change information and vegetation-related change information. Direct association of object types and radiation, texture and geometric features is established by analyzing the characteristics of the three types of objects. During the application of object-level change detection method, first, feature vectors of objects are constructed by controlling the weight of radiation, texture and geometric features in different ways; then feature vectors of objects in multi-temporal images are analyzed with the method of object change vector analysis to obtain the change information of object types that are sensitive to a certain feature. In order to verify the validity of this method, this paper uses the high-resolution remote-sensing images from the Internet captured before and after the Japanese earthquake on March 11, 2011 to conduct some change detection experiments based on multifeature integration. Damage information is extracted and by controlling the weight of features, building damage, damage caused by submergence of seawater and vegetation damage are detected respectively. Experiments show that the method and processing put forward in this paper, flexible, practical and adaptable, are effective in such applications as the extraction of information about damage caused by earthquake and tsunami, and investigation of land use change.
\end{abstract}

\section{INTRODUCTION}

When a natural disaster strikes, what can best reflect the severity of disaster damage is the things that are closely related to people's lives and property, the damage of such artificial objects as residential areas, factories, roads, and public facilities. Remote-sensing technology can assess the damage to the area affected by the disaster immediately after the disaster happens to guide disaster relief work. With the development of remotesensing technology and the frequent strikes of natural disasters in recent years, wide attention has been attracted to the application of remote-sensing technology in prevention of natural disasters and post-disaster assessment and reconstruction. With the rapid development of spatial information technology, especially the development of remote sensor with high spatial and temporal resolution, spatial information technology has played an increasingly prominent role in disaster reduction ( $\mathrm{Li}, 2008)$. Remote-sensing technology has such advantages as quick access to information, a large amount of information, different methods for information, a short update cycle and omni-directional and allweather dynamic monitoring, so it can provide a new high-tech means for earthquake prediction, earthquake damage investigation and damage assessment (Chen, 2008). In many remote-sensing applications, change detection has been an important technique used for post-disaster emergency rescue, disaster monitoring, investigation and assessment, and postdisaster reconstruction by analyzing the pre-disaster and postdisaster remote-sensing images comprehensively to obtain the information about damage to the affected areas rapidly. In addition, the technology based on change detection of multitemporal remote-sensing images has been widely used for the construction of national economy and national defenses in many fields, such as agricultural surveys, forestry monitoring, urban management and planning, basic geographic database update, land degradation and desertification monitoring, marine and inland water monitoring, coastal regional environmental monitoring, wetland monitoring and management, natural disaster monitoring, and military reconnaissance and battle damage assessment and so on.

The existing change detection methods with low detection accuracy and efficiency mainly use pixel-level processing, which depends on the limited features owned by the pixels for low-level processing and depends heavily on threshold selection. With the development of remote-sensing sensors, the resolution of images has become higher and higher. Many scholars have pointed out that in the applications of remote-sensing images with middle and high resolution it has become increasingly difficult for the pixel-based image analysis to be competent. In recent years, an object-oriented image analysis has come into being, which is also applied to change detection, called Objectlevel Change Detection, also called Object-Oriented Change Detection or Object-based Change Detection. This method has attracted international attention of scholars in related fields, and it has been considered to be the most potential method for change detection (Volker, 2004; Ai-Khudhalry, 2005; Ofer, 2005). Object-level Change Detection uses object-oriented image analysis technology, getting change information by comparing the segmentation and classification results, or extracting change information by comprehensively analyzing the objects of multi-temporal images from such aspects as spectral, texture, structure, topology, and contextual information and so on, so this method has high detection accuracy and robustness. Using GIS data as auxiliary information, Volker (Volker, 2004) has put forward a change detection method of comparison after classification, which has achieved good results. While studying the shrubs in the arid and semi-arid regions of southwestern America, Andrea (Andrea, 
2004) has put forward the method in which segmentation is conducted after classification and then change of shrub coverage is detected. According to the characteristics of the high-resolution images, $\mathrm{Li}(\mathrm{Li}, 2003)$ has studied image segmentation based on texture and neighborhood relations first, based on which supervised classification is conducted; at last, change detection is conducted with the classification results for the information on change of land coverage. Comprehensive segmentation is conducted with the multi-temporal images by Baudouin (Baudouin, 2006), and then threshold is estimated based on statistical methods and distribution of object change vectors to realize change detection of forest coverage. Detection accuracy over $90 \%$ has been achieved with SPOT-HRV images in that way. Geoffrey (Hazel, 2001) has used a competitive region growing method based on Maximum Likelihood (ML) for image segmentation, and then image background is modeled with Gaussian model and objects are extracted; Next, the segmentation results and extracted objects are organized together with a digital node model; at last, object-level change detection is completed by comparing extracted objects in the digital node models of the new and old images. AI-Khudhalry (Ai-Khudhalry, 2005) has achieved good results in detecting structural damage change information in IKONOS images by using object-oriented image segmentation and classification technology. Ofer (Ofer, 2005) extracts the objects of interest in the video image sequence with image segmentation methods, and then extracts the change objects by comparing the difference of objects between images.

It can be found from the applications of change detection that even though the same group of data has been used, different applications focus on different change information. For example, when change of land use is investigated, some spectral texture of the farmland may have changed a lot, but if land use hasn't changed, this great change can be ignored. On the contrary, if health and growing conditions of crops are to be analyzed, such spectral and texture change should be paid attention to. When the spectral texture of lake water has changed greatly because of floating water plants without changing the use of lake water, this kind of change can also be ignored. But if the lake has been filled for crop planting, this kind of change should be extracted and saved, even though there is little difference between the crops and water plants in spectral and texture features. Consequently, studies and applications show that no change detection method has been generally considered to be the best; different methods are put forward in different situations for different purposes with different applicability and limitations. With the rapid development of obtaining remote-sensing data, more and more remote-sensing data and data combinations with distinctive features have put forward many new technical requirements for change detection (Sun, 2011).

Though a general change detection method doesn't exist, a method do can be designed for as more applications as possible, and the parameters of change detection models can be controlled to get the needed results in accordance with different applications. Consequently, aimed at the characteristics of change detection of high-resolution remote-sensing images, this paper has put forward an object-level change detection method based on multi-feature integration. This method has take into account the properties of different types of object and application purposes, so it has a certain flexibility and adaptability. We will discuss this method in detail in the following parts and use it to process the data from the Japanese Earthquake on March 11, 2011 to verify the validity of this method.

\section{CHANGE DETECTION BASED ON MULTI- FEATURE INTEGRATION AND TAKING INTO ACCOUNT PROPERTIES OF DIFFERENT TYPES OF OBJECT}

The largest difference between object-level image analysis and pixel-level image analysis is that the image objects have richer and more reliable feature attributes than a single pixel. In pixellevel image analysis, pixels mainly have spectral features, while objects have reliable texture features, shape and topological features as well as spectral features. As to different types of objects, different typical features are reflected in the remotesensing images, so different kinds of feature information can be used flexibly to realize focus on a certain object type during image analysis. Consequently, though change detection applications vary, applications of high-resolution remotesensing images can be roughly divided into three categories: one, the change detection related to artificial objects; second, change detection related to water body; three, change detection related to vegetation. And the hybrid applications can still be divided into such three categories. The three applications are related to three types of objects, so by analyzing the properties of the three types of objects, constructing different feature vectors between objects and using the object change vector analysis, the properties of the three types of objects can all be taken into consideration by the change detection algorithm. Thus, the three applications can be controlled easily.

In the Pixel-level Change Vector Analysis, because the locations of pixels in the grid images are regular, positions of pixels can be found correspondingly between the images after registration. But it is different in the object-based change detection, in which if the objects of multi-temporal images are extracted by independent segmentation, objects in multitemporal images may not correspond spatially. If one-to-one correspondence doesn't exist between objects, change vector analysis can't be conducted. In order to solve this problem, multi-temporal remote-sensing images can be combined and segmented synchronously as a multi-band image. The image objects segmented in this way will surely be one-to-one corresponding spatially. Consequently, OCVA (Objects Change Vector Analysis) can be conducted. The object features used in this paper mainly consist of spectral, texture and geometric features (Sun, 2008; Chen, 2011).

\subsection{Feature Selection and Basic Concepts}

The reason why the spectral, texture and geometric features are selected in this paper is that firstly, for the image objects, what can be obtained the most easily and can be the most effective is these three types of features; in addition, in the abovementioned three applications, the difference is mainly reflected by these features, so the tendency of change detection can be controlled by controlling the weight of these features. For example, water body with large scale shows comparatively smoother imaging texture, darker radiometric brightness and regular geometric shape; artificial buildings have smaller scale with rough texture, brighter radiometric brightness and broken shape; the scale, spectrum and texture of the vegetation are between the former two, among which the farmland has comparatively more regular shape, the forests have rougher texture and grass land has smoother texture. Consequently, by controlling the roles that the features have played, the degree of concern about different types of objects can be realized. 
2.1.1 Spectrum : The spectrum here mainly refers to the gray value of each wave band of the images used for change detection. The spectral information of objects is the average gray value of each wave band within the objects. As to the random object $R$, the average gray value of this object at the wave band $\mathrm{L}$ can be calculated by the following method:

$$
\overline{C_{L}}=\frac{1}{n} \sum_{i=1}^{n} C_{L i}
$$

$\bar{C}_{L}$ shows the average gray value. $\mathrm{L}$ is the wave band; $\mathrm{n}$ is the number of pixels within the object $R$ at the wave band $\mathrm{L}$, and $C_{L i}$ is the gray value of the pixel $i$.

2.1.2 Texture : Texture feature shows the law of spatial distribution of spectral features within the image. In objectoriented image analysis, an object is the collection of pixels. When the area of the object comes to a certain degree, the internal spectral distribution will also show its unique texture property. For some images, the texture features are much more useful than the spectral features alone. If the texture is adopted as a property parameter to be involved in analysis, the accuracy of analysis will be greatly increased.

Though texture has been studied for years, no uniform definition has been given to it. In image processing, the texture is mainly be described by statistical methods, structured methods and spectral methods. The common methods for texture description and analysis are stroke length statistical method, histogram analysis, Laws texture energy measurement, GLCM (Gray Level Co-occurrence Matrix) analysis, autocorrelation function analysis, Fourier spectrum analysis, and Markov random field analysis. For the details of these methods, the documents (Rafael, 2003) can be read for reference. In this paper, the texture property of images is described in the easiest standard deviation method as follows:

$$
\sigma_{L}=\sqrt{\frac{1}{n-1} \sum_{i=1}^{n}\left(C_{L i}-\bar{C}_{L}\right)^{2}}
$$

$\sigma_{L}$ is the standard deviation of the object $R$ at the wave band of $\mathrm{L}$.

2.1.3 Geometric Feature : In remote-sensing images, the geometric features of the plane region (image entity) mainly consist of area, perimeter, position and shape. If an object is extracted in multi-temporal images, image objects at the place where change has happened show difference not only in spectrum and texture but also in geometric property. This kind of change in geometric property is more obvious when such objects as farmland, lakes and buildings have changed. Consequently, geometric features can be used in change detection to measure whether the structure of an object has changed. In addition, when multi-scale or full-scale segmentation is conducted, some objects get regular shapes, while some others get broken shapes, so the shape factor which is used to describe the regularity of the shape of objects is also one of the geometric features. Consequently, geometric feature can be used in two situations; one is dynamic geometric feature for structure analysis and the other is static geometric feature for describing the regularity of object shape.

After segmentation, as to the geometric features, not only the shapes of objects correspondingly at each wave band are identical, shapes of the objects at the corresponding positions in two- phase images are also identical. Consequently, if structural change analysis needs to be focused on, the geometric feature
$S_{i}$ here isn't the static geometric feature of the image objects after segmentation but a dynamic geometric feature, which can be obtained by the following method "a synthesis method combining independent segmentation and integrated segmentation” (Sun, 2008).

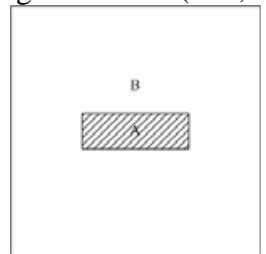

(a)

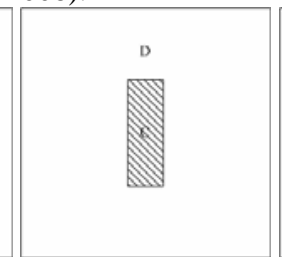

(b)

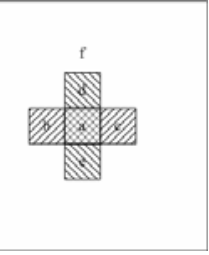

(c)
Figure.1 Diagram of Synthesis Method Combining Integrated Segmentation and Independent Segmentation, (a) Diagram of

Phase 1.(b) Diagram of Phase 2.(c) Diagram of Integrated

Segmentation of Phase 1 and Phase 2.

Shape of the object - a in Figure. 1 (c) is a static shape obtained after integrated segmentation of phase 1 and 2, and can't be used for change detection. But taking a as the original object, independent segmentation is conducted to phase 1 and 2 respectively with the parameters of integrated segmentation. Then, in phase 1, a will finally become A in Figure. 1(a), and in phase 2, a will finally segmented into $C$ in Figure. 1(b). The geometric features of $\mathrm{A}$ and $\mathrm{C}$ is what are needed by a in the algorithm put forward in this paper. When the shapes of A and $\mathrm{C}$ aren't identical, it can be judged that geometric change has happened to $\mathrm{A}$ in phase 1 , which has changed into $\mathrm{C}$.

When the static features are used, if needed, they can be defined as the synthetic value of several geometric features as shown in the Formula (3), but they can also be expressed by a certain geometric feature. In the examples given in this paper, the geometric features have mainly adopted shape vector and shape factor (Sun, 2008).

$$
S=\sum_{i=1}^{n} w_{i} \cdot S_{i}
$$

In the formula, $\mathrm{n}$ is the number of geometric features and $S_{i}$ is the geometric feature with the sequence number $i$, $\sum_{i=1}^{n} w_{i}=1$

\subsection{Algorithm}

Assume that $O_{1}$ and $O_{2}$ are two objects at the same location in the images $f_{1}$ and $f_{2}$ respectively. Their feature vectors are $p_{1}\left(p_{11}, p_{12}, \cdots, p_{1 n}\right) \quad$ and $\quad p_{2}\left(p_{21}, p_{22}, \cdots, p_{2 n}\right)$ respectively, and $n$ is the number of object features. Then the $\begin{array}{llllll}\text { difference vector of } & \mathrm{O}_{1} & \text { and } & \mathrm{O}_{2}\end{array}$ is $p_{c}\left(p_{21}-p_{11}, p_{22}-p_{12}, \cdots, p_{2 n}-p_{1 n}\right)$. So by analyzing properties of $p_{1}, p_{2}$ and $p_{c}$, such as change of angle between $p_{1}$ and $p_{2}$, it can be judged whether any change has taken place to $\mathrm{O}_{1}$ and $\mathrm{O}_{2}$ or what changes have taken place.

There are two ways for conducting multi-feature integration. The first one is that when constructing feature vector, 
$p_{1}\left(p_{11}, p_{12}, \cdots, p_{1 n}\right)$ and $p_{2}\left(p_{21}, p_{22}, \cdots, p_{2 n}\right)$ are made up by spectrum, texture and geometric feature at the same time. So the vectors $p_{1}$ and $p_{2}$ can be expressed as $p_{1}\left(g_{11}, g_{12}, \cdots, g_{1 n}, t_{11}, t_{12}, \cdots, t_{1 n}, s_{1}\right)$ and $p_{2}\left(g_{21}, g_{22}, \cdots, g_{2 n}, t_{21}, t_{22}, \cdots, t_{2 n}, s_{2}\right) ; \mathrm{n}$ is the number of image wave bands; $g_{1 i}$ and $g_{2 i}$ shows the gray values of the two-phase images at the wave band $i ; t_{1 i}$ and $t_{2 i}$ shows the texture values of the two-phase images at the wave band $\mathrm{i} ; S_{1}$ and $S_{2}$ shows the real geometric features of the current objects at two phases. In order to make comprehensive use of spectrum, texture and geometric features and adjust the roles that different features play in change detection dynamically, assign weight to the three types of features. In this way, $p_{1}$ and $p_{2}$ can be expressed as $p_{1}\left(w_{g} g_{11}, w_{g} g_{12}, \cdots, w_{g} g_{1 n}, w_{t} t_{11}, w_{t} t_{12}, \cdots, w_{t} t_{1 n}, w_{s} s_{1}\right)$ and $p_{2}\left(w_{g} g_{21}, w_{g} g_{22}, \cdots, w_{g} g_{2 n}, w_{t} t_{21}, w_{t} t_{22}, \cdots, w_{t} t_{2 n}, w_{s} s_{2}\right)^{s t a n}$ . $w_{g}, w_{t}$ and $w_{s}$ are the impact factors of spectrum, texture and geometric features respectively, and $w_{g}+w_{t}+w_{s}=1$.

The other way is using spectrum, texture and geometric, respectively construct the feature vectors $p_{1}^{g}\left(g_{11}, g_{12}, \cdots, g_{1 n}\right)$ and $p_{2}^{g}\left(g_{21}, g_{22}, \cdots, g_{2 n}\right)$, $p_{1}^{t}\left(t_{11}, t_{12}, \cdots, t_{1 n}\right)$ and $p_{2}^{t}\left(t_{21}, t_{22}, \cdots, t_{2 n}\right), s_{1}$ and $s_{2}$. In this way, each feature can be used for object change vector analysis and the final change detection result will be the integration of analysis results of the three feature change vectors.

In the two ways mentioned above, weight can be selected according to applications. For example, for detection of the flooded area, only geometric features can be used for structural comparison, or increase the weight of the texture feature for change vector analysis according to the fact that there is much difference in texture before and after flood.

\subsection{Algorithm Implementation}

When objects change vectors are obtained, the key point turns into how to identify real change objects with the change vectors. It can be conducted from two angles: one is to turn a change vector into a scalar for measuring the change intensity of objects; the other is to analyze a vector directly. The former method is adopted in this paper. Because the two images involved in change detection are segmented at the same time as two logical data layers, the scalar finally used to measure change degree of objects shows a certain logical relation between two data layers. Consequently, in this paper, the scalar used for identifying the change intensity of objects is called Inter-layer Logical Value.

In order to get the "inter-layer logical value" by analyzing the change vectors, the easiest way is to take the mode of object change vector $\left\|p_{c}\right\|$ as inter-layer logical value to measure the change intensity of the object, namely

$$
C=\left\|P_{c}\right\|=\sqrt{\sum_{i=1}^{n}\left(p_{2 i}-p_{1 i}\right)^{2}}
$$

But this method has many disadvantages. One is that when the elements in the feature vectors belong to multi-features and each feature has a different dimension with different value range, the "inter-layer logical value" obtained in this way isn't reasonable, because it is difficult to control the contribution of each kind of feature value in change detection. Because the standardization of dimensions is very complicated, in order to solve this problem, $\chi^{2}$ transformation is adopted in this paper for feature vectors to get "inter-layer logical value".

Ridd put forward the change detection algorithm based on $\chi^{2}$ transformation of pixels in 1998 (Ridd, 1998). This algorithm is applicable to all types of images. $\chi^{2}$ transformation is shown as follows:

$$
Y=(X-M)^{T} \sum^{-1}(X-M)
$$

In the formula, $Y$ is the pixel value of the output change image, and the numerical magnitude shows the change degree of pixels. $\mathrm{X}$ is the pixel change vector of bi-temporal images. $\mathrm{M}$ is the mean residual vector of the whole image at different bands. $\mathrm{T}$ stands for matrix transposition. $\sum^{-1}$ is the covariance inverse matrix of bi-temporal images at different wave bands. The role of the transformation is that $\mathrm{Y}$ is the random variable subject to $\chi^{2}$ distribution taking $\mathrm{P}$ as the freedom degree. $\mathrm{P}$ is the number of image bands. The smaller $\mathrm{Y}$ is, the less probably that the pixel has changed, and the larger $\mathrm{Y}$ is, the more probably that the pixel has changed. Consequently, the numerical magnitude of $\mathrm{Y}$ can be used to measure the change intensity of images. The numerical magnitude of $\mathrm{Y}$, obtained through $\chi^{2}$ transformation by object vector, can also be used as inter-layer logical value for measuring change degree of image objects. Consequently, in this paper this method has been improved and $\chi^{2}$ transformation based on object change vector has been put forward. The transformation formula is as follows:

$$
C=(X-M)^{T} \sum^{-1}(X-M)
$$

In the formula, $\mathrm{C}$ is the result of $\chi^{2}$ transformation of the current object change vector, also inter-layer logical value, which shows the change intensity of current object. $X$ is the change vector of the bi-temporal image object. $\mathrm{M}$ is the mean residual vector of vectors of all image objects parameters. $\mathrm{T}$ stands for matrix transposition. $\sum^{-1}$ is the covariance inverse matrix of vectors of objects parameters in bi-temporal images. Similar to pixel-based $\chi^{2}$ distribution, $\mathrm{C}$ is the random variable taking $\mathrm{n}$ as the freedom degree subject to $\chi^{2}$ distribution. $\mathrm{n}$ is the dimension of vectors of the image objects parameters. The smaller $\mathrm{C}$ is, the less probably that the image object has changed, and the larger $C$ is, the more probably that the object has changed. The advantage of $\mathrm{C}$ as inter-layer logical value is that $\chi^{2}$ distribution properties can be used directly to estimate threshold and it can extract the changed objects automatically. In addition, from pixels to objects, amount of data drops dramatically, so threshold segmentation result and change result can be obtained quickly. In this situation, even if there appear errors in automatic 
threshold selection, real-time threshold adjustment and check of results can also be realized by manual adjustment.

According to the theory of $\chi^{2}$ transformation, $\chi^{2}$ transformation is also a method for describing the degree of difference between vectors. When the covariance in Formula (6) is a unit matrix, the value of $\sqrt{C}$ is simplified into Euclidean distance. In addition, it should be paid attention to that during the object-level $\chi^{2}$ transformation, the area of the object need to be considered when each item in the covariance matrix $\mathrm{Cov}$ is used for calculation:

$$
\operatorname{Cov}=\left(\begin{array}{l}
c_{11}, c_{12}, \ldots, c_{1 n} \\
c_{21}, c_{22}, \ldots, c_{2 n} \\
\ldots \\
c_{n 1}, c_{n 2}, \ldots, c_{n n}
\end{array}\right)
$$

$\left(c_{i j}\right)^{2}=\frac{1}{M} \sum_{\text {index }=1}^{N} m_{\text {index }} \cdot\left[X_{\text {index }}(i)-M(i)\right] \cdot\left[X_{\text {index }}(j)-M(j)\right]$

In the formula, $\mathrm{N}$ is the number of all the objects. $M=\sum_{\text {index }=1}^{N} m_{\text {index }}, m_{\text {index }}$ is the area of the object at the sequence number of index.

According to the theory of $\chi^{2}$ transformation, $\chi^{2}$ transformation is also a method for describing the degree of difference between vectors, a kind of method for describing the covariance distance. Different from Euclidean distance, this method considers links between different features. And it is scale-invariant, no requirements for the dimension and scale of the vector components to be involved in transformation. Consequently, it is safe to say that standardization is conducted by $\chi^{2}$ transformation in the form of covariance matrix. The standardization has solved the problem of interaction between elements with different senses and different dimensions from theory.

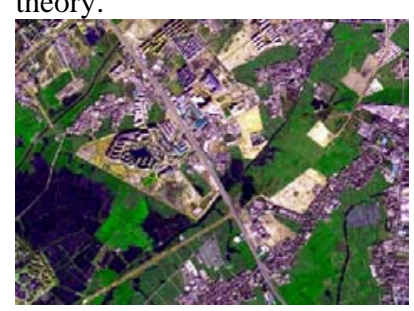

(a)

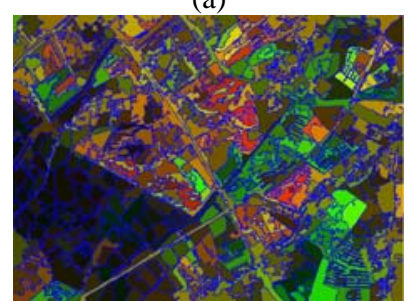

(c)

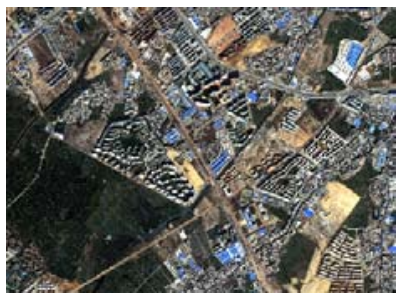

(b)

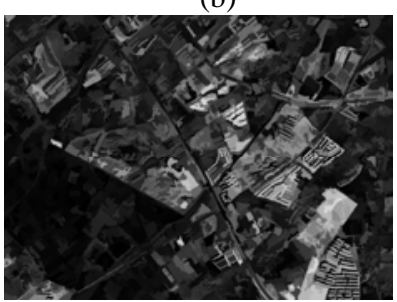

(d)

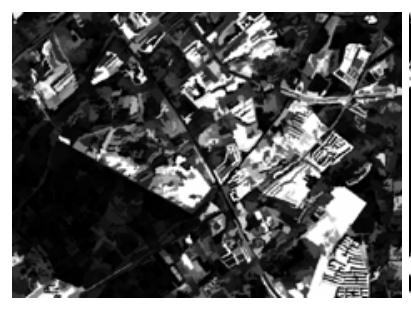

(e)

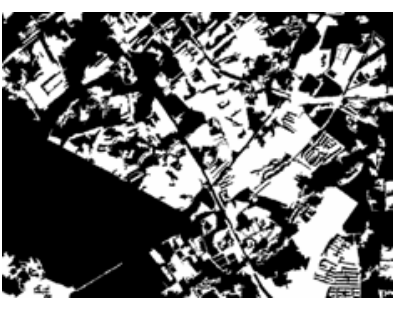

(f)
Figure.2 Examples of Change Detection with Multi-feature Synthesis (Chen, 2011), (a)Spot5 Image of Kunming in January, 2004 (b)QuikBird Image of Kunming in May, 2006, (c)Segmentation Result of a, b Image

Combination(Scale40)(d)Image Generated by Modes of Object Change Vectors, (e) Image with Inter-layer Logical Value

Obtained by $\chi^{2}$ Transformation,(f) Change Result of Segmentation with Slack Iteration Method to image (e)

It can be seen form Figure. 2 (a), (b) that the two-phase images used for change detection are captured by different sensors, so the radiometric properties of the two images are different in some ways; especially water and vegetation are quite different in spectrum. In view that the reduce or increase of artificial buildings caused by urban construction is paid attention to, in the multi-feature synthesis method, the weight of texture feature is heavier than that of the spectral feature, and that of the geometric feature is 0 . It can be seen from the result that though there has been much difference in the color of the lake water and vegetation, still no change is considered.

\section{APPLICATION OF OBJECT-LEVEL CHANGE DETECTION IN JAPANESE EARTHQUAKE ON MARCH 11, 2011}

Japan was struck by a magnitude 9.0 earthquake near its east coast of Honshu Friday, March 11, 2011 at 01:46:23 PM Beijing. The epicenter was located in the Pacific Ocean east of Miyagi-ken, and the focal depth was $10 \mathrm{~km}$. The earthquake was even strongly felt in Tokyo. Influenced by this earthquake in the west of Pacific Ocean, a devastating tsunami hit the coast of Iwate- ken, Miyagi-ken and Fukushima- ken, which resulted in extensive damage, heavy casualties and many missing people. Many towns have been flattened by the tsunami. Several coastal cities were flooded by the seawater, and public facilities, houses and cars were damaged to varying degrees. What is worse, the earthquake resulted in nuclear leak from Fukushima I Nuclear Power Plant, which has great impact on the environment of Japan and neighboring countries and regions.

Change detection based on remote-sensing images can be very effective in acquiring information on damage caused by disaster quickly. But after the earthquake and tsunami, very limited first-hand data can be obtained in China, so we have mainly downloaded some remote-sensing pictures of some areas before and after the disasters from some major news sites to compare and analyze them. In the following part, some downloaded pictures are processed with the multi-feature integration objectlevel change detection put forward in this paper to verify the validity of this method. Data about the Japan earthquake in this paper are from Australian Broadcasting Corporation (ABC News) (ABC, 2011).

\subsection{Data Pre-processing}

For change detection, different-phase images involved in operation must correspond spatially, which means that image registration is necessary. In addition, if images are captured in different seasons or at different temporal, or such external 
conditions as sun elevation angle, cameral angle, and weather conditions are different, or coverage of cloud, rain or snow is different, image radiometric will also be different. Consequently it is necessary for most change detection methods to eliminate the non-change radiometric difference between images first with radiometric correction methods.

3.1.1 Geometric Pre-processing : When data are available, geometric pre-processing need to be carried out first, because the remote-sensing data before and after a disaster are often with inaccurate coordinates or lack of coordinate information. As to the geometric pre-processing for change detection, it is generally required that ortho-rectification should be conducted to the two-phase images respectively to promote change detection accuracy. However, ortho-rectification has strict requirements for data sources. Consequently, the most common method used is to implement relative registration between the two-phase images. Because a large disaster may cause largescale changes to the ground, it is often difficult to match the corresponding points automatically. In addition, if there has been much difference in cameral angles between the remotesensing images, it can also be difficult to match the corresponding points automatically. If automatic matching of corresponding points is difficult for the multi-temporal data, registration of them can be implemented by combing artificial point selecting and automatic matching.

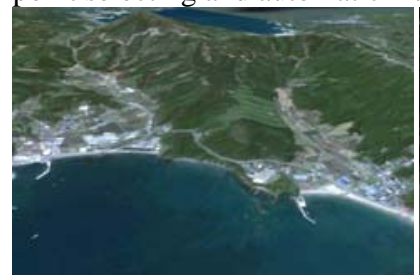

(a)

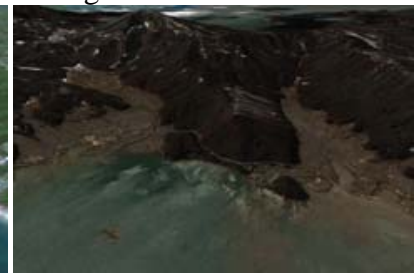

(b)

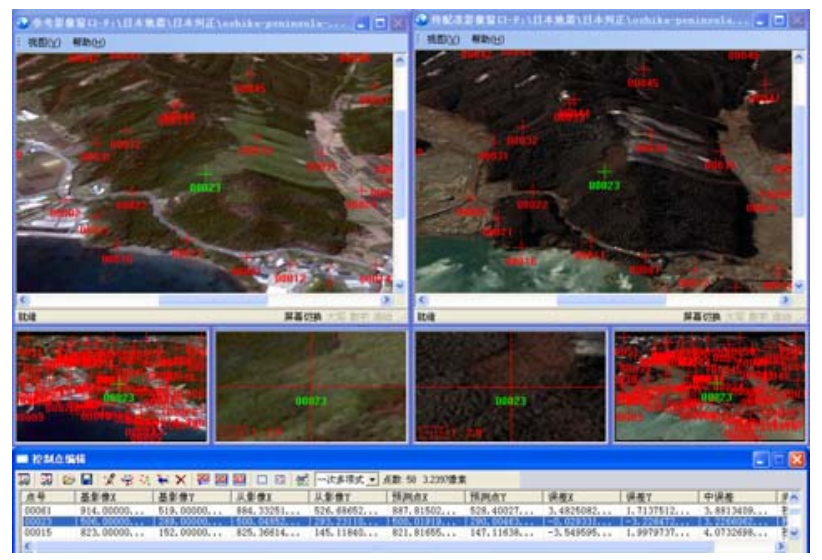

(c)

Figure.3Geometric Pre-processing, (a)Yagawahama in Miyagi Before Disaster (b)Yagawahama in Miyagi After Disaster, (c) Interface of Image Registration with SIFT Automatic Matching

As shown in Figure.3, large angle pictures (three- dimensional rendering screenshots) of Yagawahama in Miyagi before the disaster and after the disaster are obtained from the Internet. In this paper, corresponding points are located with SIFT matching and registration between images is implemented by the correction method based on triangulation.
3.1.2 Radiometric Pre-processing : Change on the ground is reflected in the remote-sensing images in the form of change of such radiometric features as spectrum and texture. However, not all the radiometric difference in the two-phase images has been caused by change on the ground. Consequently, it is necessary to conduct radiometric correction to eliminate nonchange radiometric difference before change detection.

Radiometric correction refers to eliminate or weaken the inconsistence between the sensor measurements and the spectral reflectance or spectral radiance of objects. There are absolute radiometric correction and relative radiometric correction. Absolute radiometric correction mainly corrects the distortion of radiation irrelevant to the change of radiometric features on the surface of objects. Such distortion of radiation is mainly caused by status and position of sensor, the sun light and atmospheric diffusion and absorption. It is difficult to realize absolute correction, so relative radiometric correction is commonly used to make the radiance distribution of the multitemporal images involved in change detection comparatively consistent. However, most relative radiometric correction methods are aimed at the same sensor and it should be assumed that radiance distribution between images conforms to linear relationship. And in applications, the radiometric properties of quite many multi-temporal images captured by the same sensor don't show obvious linear relationship, and the linear relationship of images captured by different sensors is even less obvious.

In order to solve the problem mentioned above, Sun (Sun, 2010) put forward the method for treatment of relative radiometric consistency based on Wavelet Transform and Low-pass Filter (WLPF). This method processes the low-frequency part of an image without affecting the high-frequency part. In this method, the relative radiometric consistency processing based on lowpass filter is only applied to the low-frequency part after wavelet transform, and wavelet reverse transform is conducted after that to get the result image. Overall brightness and color distribution of the reference image is used to replace that of the image to be corrected so that radiometric properties has been adjusted and image texture has been protected at the same time. This method is especially suitable for the radiometric correction of the nonlinear differential images. The results of this algorithm are shown as follows in Figure. 4.

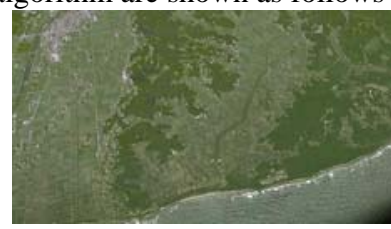

(a)

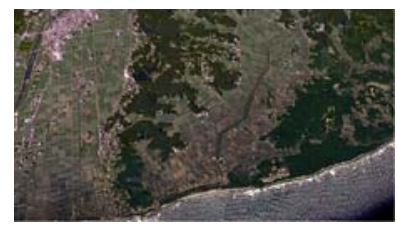

(c)

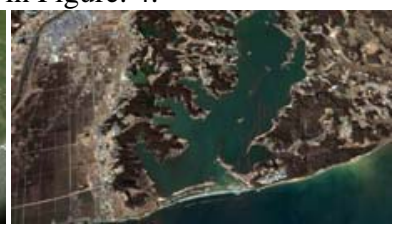

(b)

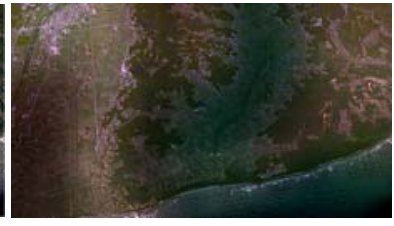

(d)
Figure.4 Comparison of Radiometric Pre-processing to Data of Kashimaku in Minamisoma, Japan before and after Disaster, (a)Kashimaku in Minamisoma before Disaster (b)Kashimaku in Minamisoma after Disaster, (c)Relative Radiometric Correction Based on Mean- Variance (d)Relative Radiometric Correction Based on Wavelet Transform and Low-pass Filter. 
It can be seen from Figure. 4 that because of the principle and scale, after the original image is processed with the method of wavelet transform and low-pass filter, compared with (c), distortion happens as shown in (d). But such radiometric processing can improve the detection accuracy because the result has removed the radiometric difference caused by nonchange information.

Relative radiometric correction shown in Figure. 4 should be on the premise that changed area in the image is much smaller than the proportion of the unchanged area.

3.2 Object Extraction Based on Multi-scale/ Full- scale Segmentation

For object-level change detection, an image must be turned into a set of objects and image segmentation can be used to realize this. Different purpose and different image resolution of objectlevel image analysis will result in different scales needed to extract image objects.

Multi-scale and full-scale segmentation has been used for extracting objects in this paper (Sun, 2009). The segmentation results of the integrated images of Fujitsuka in Sendai before and after the disaster on different scales are shown in the following part.

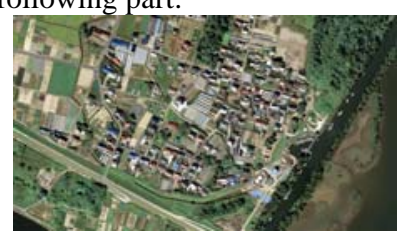

(a)

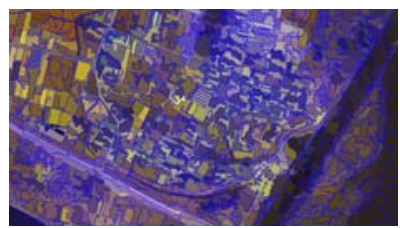

(c)

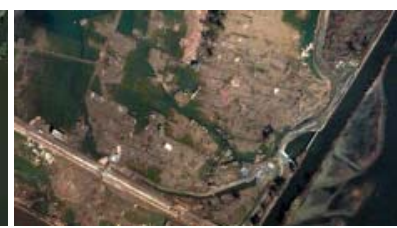

(b)

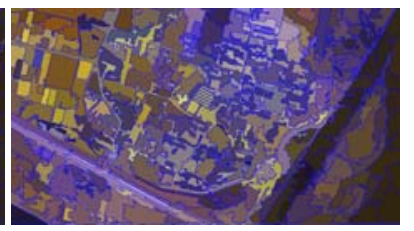

(d)
Figure.5 Instances of Multi-scale Segmentation, (a)Fujitsuka in Sendai before Disaster,(b)Fujitsuka in Sendai after Disaster, (c)Integrated Segmentation Result on Scale 20 (d)Integrated Segmentation Result on Scale 40

Figure. 5 shows the integrated segmentation results of the images before and after the disaster. Object change vector analysis can be used to extract changed area based on (c) and (d). If comparison after classification or comparison after feature extraction is used, then multi-scale or full-scale segmentation can be conducted to extract image objects or specific objects of the two-phase images respectively, after which change detection follows.

\subsection{Extraction of Damage Information with Change Detection}

After geometric preprocessing, radiometric preprocessing and image object extraction, change information caused by the disaster can be extracted according to different applications.

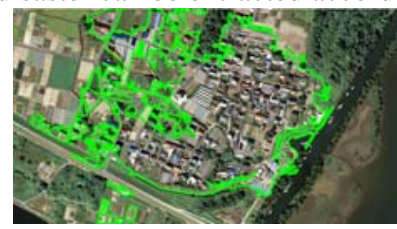

(a)

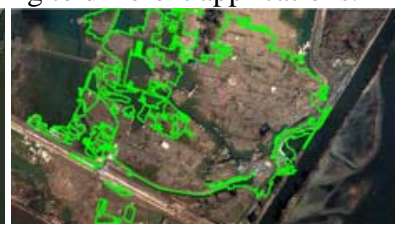

(b)

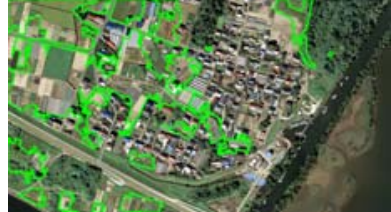

(c)

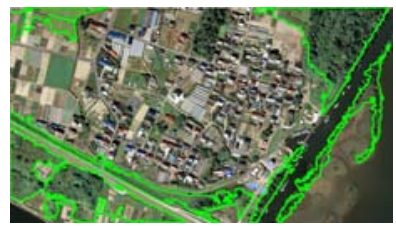

(e)

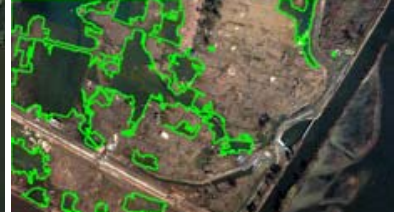

(d)

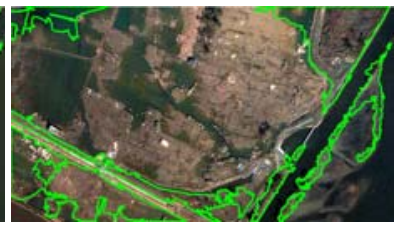

(f)
Figure.6 Extraction of Building Damage, Submerged Conditions and Vegetation Damage with Multi-feature synthesis method, Green Polygon Area being Changed Area, (a)Building-related Change Detection Result of Superimposed Images before Disaster,(b)Building-related Change Detection Result of Superimposed Images after Disaster, (c)Water-related

Change Detection Result of Superimposed Images before

Disaster(d)Water-related Change Detection Result of Superimposed Images after Disaster, (e)Vegetation-related Change Detection Result of Superimposed Images before

Disaster,(f)Vegetation -related Change Detection Result of Superimposed Images after Disaster.

Just as shown in Figure.6, with the method put forward in this paper, by controlling spectrum, texture and geometric features and using the integrated segmentation results in Figure.5 (c), (d), change information related to buildings, water body and vegetation has been extracted respectively. During implementation, change information related to buildings is extracted by increasing weight of texture features and static shape features; change information related to water body is extracted by controlling texture features and dynamic shape features; change information related to vegetation is extracted by controlling spectrum and texture. It can be seen from Figure. 6(e), (f) that when detecting vegetation-related changes, geometric feature hasn't been used. Consequently, change of the farmland in the upper left hasn't been detected for texture and radiometric changes are not obvious. In order to improve the accuracy of detecting farmland, weight of dynamic geometric feature can be increased.

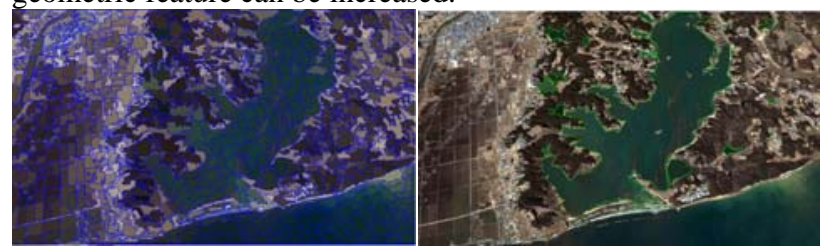

(a)

(b)

Figure.7 Extraction of Submerged Area in Figure.5 with Change Detection Technology, (a) The result of before-post images segmentation, (b) The result of stagnant water area extraction.

Figure. 7 shows the change detection result with the data processed as shown in Figure.4, extracting the submerged stagnant water area of Kashimaku in Minamisoma. It can be calculated from the detected result that submerged area may be about 190, 000 square meters. In view that radiometric preprocessing has been conducted to the two images, even without weight redistribution, it is the texture feature that 
matters. In fact, texture feature has been mainly used in this instance.

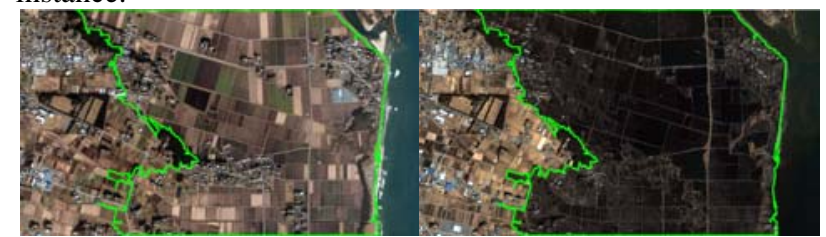

(a)

(b)

Figure.8 Instance of Extracting Watered Area Swept by Tsunami, (a)Haranomachi in Minamisoma before

Tsunami(b)Haranomachi in Minamisoma after Tsunami. Figure. 8 shows the instance of extracting watered area swept by the tsunami with the method put forward in this paper. The green polygon area is the change caused by tsunami. The remote-sensing images of this area before and after the tsunami are almost the same in radiometric property, and there has been little difference in texture and structure. Consequently, during processing, tsunami-affected area has been extracted by increasing the weight of spectral feature.

In addition, in Figure. 8 the changed area in the image is too large in proportion. In order to prevent damage to the radiometric property of the changed area, relative radiometric correction hasn't been conducted.

\section{CONCLUSION}

In all the change detection applications based on remotesensing images, different purposes of application focus on different change information, so there is no universal change detection method. Aimed at such characteristic, this paper has summarized the change types during change detection and has managed the object features, based on which a multi-feature integrated object-level change detection method considering purposes of applications and properties of different types of object has been put forward. Based on feature vector analysis, this method detects changes of different object types by constructing feature vectors to the different features of image objects with different strategies and different weights. Thus this change detection method has better adaptability. By detecting and assessing the data from Japanese earthquake on March 11, 2011, it shows that the method put forward in this paper can applied to change detection of different types of object by controlling parameters, so it has some flexibility, practicality and adaptability.

\section{Acknowledgements}

This work is supported by National Natural Fund of China (NSFC) (No.40901211, No. 61001187, No.60602013, No. 41101414)

\section{References}

Ai-Khudhalry D. H. A., 2005, Caravaggii I, Giada S. Structural damage assessments from Iknos data using change detection, Object-level Segmentation, and Classification Techniques. Photogramm Eng Rem, 71(7): 825-837

Andrea S. L., Albert R., Kris M. H., et al., 2004, Objectoriented image analysis for mapping shrub encroachment from 1937 to 2003 in southern New Mexico. Remote Sens Environ, 93: $198-210$

Baudouin D., Patrick B., Pierre D., 2006, Forest change detection by statistical object-based method. Remote Sensing of Environment, 102:1-11

Chen W. K., He S. L., Zhang J. F., et al., 2008, A Review on Methods of Extracting Earthquake Damage Information Using
Remote Sensing Technology, Northwestern Seismological Journal, 30(3): 88-93

Chen Y., Sun K. M., 2011, A new Method of Change Detection, 2011 International Conference on Information Science and Engineering, V(3),1544

Hazel G. G., 2001, Object-Level Change Detection in Spectral Imagery, IEEE TRANSACTIONS ON GEOSCIENCE AND REMOTE SENSING, 39(3):553-561.

Li D. R., Chen X. L., Cai X. B., Spatial Information Techniques in Rapid Response to Wenchuan Earthquake, Journal of remote sensing, 2008,12(6):841-851

Li J. C., Qian S. M., Chen X., Object-Oriented Method of Land Cover Change Detection Approach Using High Spatial Resolution Remote Sensing Data, IEEE, 2003

Ofer M., Arie P., Amir A., Objects based change detection in a pair of gray-level images. Pattern Recognition, 2005, 38, 19761992

Ridd M K and Liu J J. A comparison of four algorithms for change detection in an urban environment, Remote Sens Environ, 1998, 65(2): 95-100

Rafael C. Gonzalez, Richard E. Woods. Digital image processing(Second. Edition), Publishing house of electronics industry, 2003, 522-539

Sun X. X., Zhang J. X., Yan Q., et al., A Summary on Current Techniques and Prospects of Remote Sensing Change Detection, . Remote Sensing Information,2011(1): 19-123

Sun K. M., Object-Oriented Change Detection of Terrain Objects, Wuhan University, 2008: 17-20

Sun K. M., Li D. R., An Object-oriented Image Smoothing Algorithm Based on the Convexity Model and Multi-scale Segmentation, Geomatics and Information Science of Wuhan University, 2009, 423-487.

SUN K. M., SUI H. G., et al., A New Relative Radiometric Consistency Processing Method For Change Detection Based on Wavelet Transform and Low-pass Filter, SCIENCE IN CHINA,2010.

Volker W., Object-based classification of remote sensing data for change detection. Photogramm Eng Rem, 2004, 58:225-238

ABC, 2011, http://www.abc.net.au/news/events/japan-quake2011/beforeafter.htm 